Please quote as: Lehmann, K.; Oeste, S.; Janson, A.; Söllner, M. \& Leimeister, J. M. (2015):

Flipping the Classroom - IT-unterstützte Lerneraktivierung zur Verbesserung des Lernerfolges einer universitären Massenlehrveranstaltung. In: HMD Praxis der Wirtschaftsinformatik, Ausgabe/Number: 1, Vol. 52, Erscheinungsjahr/Year: 2015. Seiten/Pages: 81-95 (DOI: 10.1365/s40702-014-0102-4). 


\title{
Flipping the Classroom - IT-unterstützte Lerneraktivierung zur Verbesserung des Lernerfolges einer universitären Massenlehrveranstaltung
}

\author{
Katja Lehmann • Sarah Oeste • Andreas Janson • Matthias Söllner • \\ Jan Marco Leimeister
}

Eingegangen: 31. Oktober 2014 / Angenommen: 13. Dezember 2014 / Online publiziert: 24. Dezember 2014 (C) Springer Fachmedien Wiesbaden 2014

Zusammenfassung Durch steigende Studierendenzahlen prägen universitäre Massenlehrveranstaltungen nach wie vor das Bild in der deutschen Hochschullandschaft. Diese Lehrveranstaltungen sind gekennzeichnet durch hohe Anonymität und einen Mangel an Interaktion und Zusammenarbeit. Lernende bleiben sich im Lernprozess weitestgehend selbst überlassen und sind eher Konsumenten der Lerninhalte anstatt selbst im Mittelpunkt der Lernaktivitäten zu stehen. Jedoch bilden vor allem verschiedene Formen der Interaktion entscheidende Faktoren für Lernerfolg und Zufriedenheit eines Einzelnen. Der Flipped Classroom stellt eine Möglichkeit dar, den Nachteilen von universitären Massenlehrveranstaltungen zu begegnen. Der vorliegende Beitrag präsentiert ein IT-gestütztes Flipped Classroom-Konzept am Beispiel einer Wirtschaftsinformatik-Grundlagenveranstaltung. Das Konzept unterteilt die Veranstaltung in vier aufeinander ablaufende Lernphasen: Selbstlernphase, PeerLernphase, Transferphase und Vertiefungsphase. Durch die Integration verschiedener Lernmethoden wird sichergestellt, dass zum einen die Potentiale des IT-gestützten Lernens - beispielsweise orts- und zeitunabhängiges Lernen - adressiert und zum anderen die verschiedenen Formen von Interaktion realisiert werden. Durch die intensive Beschäftigung und den intensiven Austausch mit den Lerninhalten wird sichergestellt, dass alle kognitiven Lernzielebenen adressiert und überprüft werden. Der praktische Beitrag zeigt wie Massenlehrveranstaltungen durch Lernmethoden bereichert werden können. Zudem wird als theoretischer Beitrag die ak-

K. Lehmann $(\bowtie) \cdot$ S. Oeste $\cdot$ A. Janson · M. Söllner · J. M. Leimeister Universität Kassel, FG Wirtschaftsinformatik,

Pfannkuchstr. 1, 34121 Kassel, Deutschland

E-Mail: katja.lehmann@uni-kassel.de

M. Söllner · J. M. Leimeister

Universität St.Gallen, Institut für Wirtschaftsinformatik (IWI-HSG),

Müller-Friedberg-Strasse 8,

9000 St. Gallen, Schweiz 
tive Einbindung von Lernenden in den Lernprozess und deren Rolle als Prosument betont. Das Konzept sowie die Ergebnisse des vorgestellten Beitrages lassen sich auf andere Veranstaltungen im universitären Kontext oder die betriebliche Weiterbildung übertragen.

Schlüsselwörter Lernziele · Lernerfolg · Universitäre Massenlehrveranstaltung · Interaktion · Peer Learning · Theoriemotiviertes Design

\section{Problemstellung und Motivation}

Universitäre Massenlehrveranstaltungen, in denen ein Dozent weit über 100 Lernende lehrt, sind nach wie vor Standard in der deutschen Hochschullandschaft (Leidenfrost et al. 2009). Diese Massenlehrveranstaltungen sind gekennzeichnet durch hohe Anonymität sowie durch einen Mangel an Interaktion - nicht nur zwischen den Lernenden und dem Dozenten, sondern auch zwischen den Lernenden untereinander (Kerres 2001). Dies resultiert häufig in ungenügenden Lernergebnissen und unzufriedenen Lernenden (Lehmann und Söllner 2014). Diese Entwicklung ist höchst unbefriedigend, denn fundamentale Elemente im Lernen sind u. a. seine Meinung zu den Lerninhalten mit anderen auszutauschen und zu diskutieren (Picciano 2002).

Damit Lernende die Lerninhalte in ihrer Komplexität verstehen, diese anwenden, bewerten und mit anderen Lerninhalten verknüpfen, sollen sich die Lerninhalte in Vermittlung und Überprüfung an den kognitiven Lernzielebenen ausrichten. Nach Anderson et al. (2001) werden folgende kognitive Lernzielebenen unterschieden: Erinnern, Verstehen, Anwenden, Analysieren, Beurteilen und Gestalten. Besonders die Vermittlung und Überprüfung hoher Lernzielebenen (Anwenden bis Gestalten) erfordert ein didaktisches Konzept, welches den Lernenden in den Mittelpunkt des Geschehens rückt und verschiedene Formen der Interaktion ermöglicht. Das reine Konsumieren von Lerninhalten ist dafür nicht ausreichend. Aber besonders durch den hohen Zeit-, Ressourcen- und Interaktionsaufwand sind Vermittlung und Überprüfung hoher Lernzielebenen in traditionellen Massenlehrveranstaltungen kaum realisierbar und finden daher nur in kleinen Seminaren Anwendung. Dies führt wiederum dazu, dass Lernende die Lerninhalte in traditionellen Massenlehrveranstaltungen nicht in ihrer Komplexität anwenden, analysieren und bewerten. Notwendig wäre dafür die kontinuierliche Auseinandersetzung mit den Lerninhalten allein oder in der Gruppe, Feedbackmechanismen zur Unterstützung des Lernprozesses sowie die Interaktion und Zusammenarbeit mit anderen Lernenden (den sog. Peers) (Boud und Falchikov 2007). Die Interaktion ist ein wichtiger Faktor im Lernprozess (Moore et al. 1996), der gleichzeitig Prädiktor für den Lernerfolg ist und die Zufriedenheit der Lernbeteiligten positiv und langfristig beeinflusst (Picciano 2002). Ziel muss damit sein, den Lernenden in Massenlehrveranstaltungen in den Mittelpunkt der Aktivitäten zu rücken. Dazu gilt es die Potentiale von Kleingruppenveranstaltungen aufzugreifen und mit einem vertretbaren Zeit- und Ressourcenaufwand skalierbar auf großzahlige Veranstaltungen zu übertragen.

Geleitet von der Erkenntnis, dass Interaktion ein wesentlicher Prädiktor von Lernerfolg ist, bildet die Interaktionstheorie eine wichtige Basis zur Entwicklung eines 
skalierbaren, didaktischen Konzepts für universitäre Massenlehrveranstaltungen, das bei Beteiligten zu Lernerfolgssteigerungen führt. Ausgehend davon liegen im vorliegenden Beitrag folgende Grundannahmen zugrunde: Durch Interaktion kann der individuelle Lernerfolg gesteigert werden. Die verschiedenen Formen von Interaktion (Lerner-Lerner, Lerner-Inhalt, Lerner-Dozent), unterschieden nach Moore (1989), ermöglichen zusätzlich das Adressieren aller kognitiven Lernzielebenen im Lernprozess. Auf diese Weise wird ein umfassender und vielfältiger Austausch mit komplexen Lerninhalten möglich.

Ein innovatives Konzept, um auch in Massenlehrveranstaltungen die Lernenden verstärkt zu aktivieren, Interaktion zu integrieren sowie die Lerninhalte über alle Lernzielebenen zu vermitteln, bietet ein IT-gestütztes Flipped Classroom-Konzept. Im Flipped Classroom, auch bekannt als Inverted Classroom (Strayer 2012) oder umgedrehter Unterricht (Behringer 2014), eignen sich die Lernenden die Lerninhalte im Selbststudium zu Hause an. Dadurch erscheinen die Lernenden in der Präsenzveranstaltung mit dem Grundlagenwissen und es wird wertvolle Zeit für Fragen und Diskussionen sowie für die Anwendung des im Selbststudium erworbenen Wissens frei (Strayer 2012; Behringer 2014). Über online verfügbare Lernmaterialen, bspw. Lehrvideos und Skripte, eignen sich die Lernenden das Wissen selbständig an. Leistungskontrollen in Form von automatisch auswertbaren Tests bieten Lernenden die Möglichkeit das erlernte Wissen selbstgesteuert zu überprüfen. In die Präsenzphase werden Übungsaufgaben integriert, die per se komplexer sind und mehr auf die Anwendung, Analyse und Diskussion der Lerninhalte fokussieren (Keengwe et al. 2014). Die Präsenzveranstaltung kann durch Gruppen- und Einzelaktivitäten, Diskussionen und andere lernerzentrierte Aktivitäten gestaltet werden, um die Lerninhalte durch wichtige Verständnis- und Vertiefungsfragen zu festigen (Johnson et al. 2014), aber auch kritisches Denken, Reflexions- und Kommunikationsfähigkeiten zu schulen (Garrison und Kanuka 2004). Darüber hinaus müssen die beiden Phasen (Online und Präsenz) durch Übungsaufgaben ineinandergreifen (Strayer 2012). Ein gutes Flipped Classroom-Konzept verspricht bessere Lernleistungen und unterstützt die Interaktion und Lernmotivation während des Lernprozesses (Strayer 2012).

Vom Flipped Classroom sind zwei verwandte Konzepte abzugrenzen, welche sich in der Praxis des IT-unterstützten Lernens hoher Beliebtheit erfreuen (Martin 2012): massive open online courses (MOOCs) und small private online courses (SPOCs). MOOCs sind etablierte Kurse, die allen Menschen mit einem gemeinsamen Interesse weltweit zugänglich sind (Wulf et al. 2014). Sie richten sich an einen breiten Interessentenkreis und sind in der Teilnehmerzahl nicht begrenzt. Das Lehrkonzept ist rein online basiert, sodass alle Lerninhalte und Interaktionen auf den virtuellen Raum beschränkt sind (Haggard 2013). Im Vergleich zu MOOCs ist der Zugang zu SPOCs eingeschränkt. Solche Kurse sind nur für einen vordefinierten Personenkreis offen. Der Kurs selbst ist aber auch wie ein MOOC rein onlinebasiert. Der Flipped Classroom beinhaltet die Onlinekomponenten von MOOCs und SPOCs zum Selbststudium (Martin 2012) sowie die private Komponente der SPOCs. Zusätzlich verfügt der Flipped Classroom über wertvolle Präsenzphasen und unterscheidet sich dadurch deutlich von MOOCs und SPOCs.

Der vorliegende Beitrag beschreibt ein skalierbares IT-gestütztes Flipped Classroom-Konzept am Beispiel einer Wirtschaftsinformatik-Grundlagenveranstaltung, 
um Lernende in Massenlehrveranstaltungen über alle Interaktionsformen zu aktivieren, alle kognitiven Lernzielebenen zu adressieren und zu überprüfen und dadurch den Lernerfolg zu verbessern. Die Entwicklung des Flipped Classroom-Konzepts basiert auf dem Design Science Research Ansatz nach Peffers et al. (2006). Um sicherzustellen, dass das Flipped Classroom-Konzept die drei Formen der Interaktion berücksichtigt, wird ein Theory-driven Design Ansatz nach Briggs (2006) verfolgt. Die Grundlage bildet die Interaktionstheorie, auf deren Basis Anforderungen abgeleitet werden. In Anlehnung daran werden Designelemente hergeleitet, um in Massenlehrveranstaltungen Interaktion und alle kognitiven Lernzielebenen zu adressieren und infolgedessen Lernerfolg zu steigern. Der erste Schritt im Design Science Research Ansatz nach Peffers et al. (2006) zielt auf die Problemstellung und Motivation ab, die im ersten Kapitel erläutert wird. Kap 2 des vorliegenden Beitrages formuliert Anforderungen, abgeleitet aus der Interaktionstheorie. In Kap 3 werden die Designelemente für ein IT-gestütztes Flipped Classroom-Konzept hergeleitet, bevor im vierten Kapitel das in der Wirtschaftsinformatik-Grundlagenveranstaltung eingesetzte Flipped Classroom-Konzept dargestellt wird. Die Erkenntnisse aus diesem Einsatz werden in Kap 5 diskutiert und Handlungsimplikationen abgeleitet. Der Beitrag schließt mit einem kurzen Fazit sowie einem Ausblick auf künftige Forschungsarbeiten.

\section{Ableitung von Anforderungen aus der Interaktionstheorie}

Die Interaktion beschreibt die Interrelation zwischen Menschen und ihren kommunikativen Handlungen untereinander (Bryant und Heath 2000). Für die Verwendung von Interaktion im Umfeld Lernen trifft Moore (1989) die deutlichste Unterscheidung für den Austausch. Unterschieden wird die Lerner-Lerner-, die Lerner-Dozenten- sowie die Lerner-Inhalts-Interaktion, die für vorliegenden Beitrag übernommen wird.

Die Interaktion zwischen Lernenden und Dozenten gilt als sehr bedeutend unter den Faktoren, die das Lernen beeinflussen. Lernende, die mit dem Dozenten interagieren, sind im Lernprozess aktiver engagiert (Wang et al. 1990) und erzielen bessere Lernergebnisse. Die klassischste Form der Interaktion ist das Frage-Antwort-Spiel zwischen Lernenden und Dozenten. Der Dozent kann die Lernenden aktiv in die Lehre einbinden, den Lernfortschritt durch die Antworten der Lernenden überprüfen sowie ein direktes Feedback geben. Die Lernenden haben die Möglichkeit ihre Ideen und Gedanken einzubringen und somit neue Denkanstöße zu liefern (Gagné et al. 1993). Die Interaktion zwischen Lernenden untereinander ermöglicht einen direkten Austausch im Lernprozess. Dies fördert die Reflexionsfähigkeit der Lernenden und erhört gleichzeitig die Motivation zum Lernen (Morgan 1991). So erhalten die Lernenden das Gefühl, Teil einer Gemeinschaft zu sein und fühlen sich in ihrem Lernprozess nicht isoliert. Die Lerner-Lerner-Interaktion baut weiterhin die Anonymität untereinander $\mathrm{ab}$ und ermuntert Lernende, sich aktiv einzubringen. Die Lerner-Inhalts-Interaktion erfolgt durch die zur Verfügung gestellten Lernmaterialien. Eine Studie konnte zeigen, dass Lernende von einem hohen Interaktionsgrad profitieren und höhere Lernergebnisse erreichen (Metzger und Schulmeister 2004). Die interaktive Gestaltung von Lehr-Lern-Szenarien fördert Motivation und Aufmerksamkeit 
Tab. 1 Anforderungen aus der Interaktionstheorie

\begin{tabular}{|c|c|c|}
\hline Interaktion & Beschreibung & Anforderungen (A) \\
\hline $\begin{array}{l}\text { Lerner-Lerner- } \\
\text { Interaktion }\end{array}$ & $\begin{array}{l}\text { Lernende benötigen im Lernprozess die } \\
\text { Möglichkeit, sich mit den Peers auszu- } \\
\text { tauschen und zu diskutieren (Alavi et al. } \\
\text { 2002), um Motivation und Lernerfolg zu } \\
\text { steigern (Fredericksen et al. 2000). Bei der } \\
\text { Kollaboration lernen sie voneinander und } \\
\text { eignen sich Wissen an (Topping 2005) }\end{array}$ & $\begin{array}{l}\text { A1) Lernende sollten gemeinsam Lern- } \\
\text { material erstellen } \\
\text { A2) Lernende sollten miteinander } \\
\text { diskutieren }\end{array}$ \\
\hline $\begin{array}{l}\text { Lerner-Dozen- } \\
\text { ten-Interaktion }\end{array}$ & $\begin{array}{l}\text { Dozenten leisten Hilfestellung, geben } \\
\text { Feedback und behalten einen Überblick } \\
\text { über die Leistungen der Lernenden (Bligh } \\
\text { 1998). Bei dieser Interaktion klären die } \\
\text { Lernbeteiligten Verständnisschwierigkeiten } \\
\text { (Thurmond und Wambach 2004) }\end{array}$ & $\begin{array}{l}\text { A3) Lernende sollten Feedback } \\
\text { erhalten. } \\
\text { A4) Lernende sollten Feedback geben. } \\
\text { A5) Lernende sollten die Möglichkeit } \\
\text { haben, Verständnisfragen zu stellen }\end{array}$ \\
\hline $\begin{array}{l}\text { Lerner-Inhalts- } \\
\text { Interaktion }\end{array}$ & $\begin{array}{l}\text { Diese Form zeichnet sich durch Auseinan- } \\
\text { dersetzung mit den Lerninhalten und durch } \\
\text { Teilnahme an Diskussionen aus (Jiang } \\
\text { und Ting 2000). Übungsaufgaben, welche } \\
\text { die Lerninhalte adressieren, sollten in das } \\
\text { Lehr-Lern-Szenario integriert werden } \\
\text { (Moore und Kearsley 2011) }\end{array}$ & $\begin{array}{l}\text { A6) Lernende sollten Übungsaufgaben } \\
\text { zur individuellen Lösungserarbeitung } \\
\text { erhalten. } \\
\text { A7) Lernende sollten Übungsaufgaben } \\
\text { erhalten, um diese untereinander zu } \\
\text { diskutieren }\end{array}$ \\
\hline
\end{tabular}

bei den Lernenden und trägt zu einem erweiterten Austausch der Lernenden untereinander bei (Sims 2003).

Um Lernende in Massenlehrveranstaltungen zur Interaktion zu bewegen und somit einen lernerzentrierten Ansatz zu verfolgen, ist die Integration von Lernmethoden notwendig. Zur Adressierung der Interaktion in Massenlehrveranstaltungen werden in einem ersten Schritt Anforderungen aus der Theorie abgeleitet und formuliert. Die Ausgangsbasis für die Anforderungen bilden die drei Formen der Interaktion nach Moore (1989), deren Integration in die Lehre die Lerneraktivierung verfolgt. Die (Tab. 1) zeigt die Anforderungen (A) entsprechend der Interaktionsformen. Diese Anforderungen bilden die Ausgangsbasis für die Gestaltung des Flipped Classroom-Konzepts.

\section{Designelemente für lernerzentrierte Flipped Classroom-Konzepte}

Werden zur Adressierung der Interaktion alle Formen im Lehr-Lern-Szenario integriert, resultiert daraus die Anwendung verschiedener didaktischer Prinzipien, die v. a. den Lernmethoden von Peer Learning und Self-Assessment zuzuordnen sind. Für die theoriegeleitete Herleitung von Designelementen für ein Flipped ClassroomKonzept werden im Folgenden zunächst überblicksartig die grundlegenden Lernmethoden dargestellt. Anschließend werden die Designelemente basierend auf den Anforderungen der Interaktionstheorie hergeleitet, die als gestalterische Grundlage für ein Flipped Classroom-Konzept dienen (Tab. 2).

Peer Learning adressiert das Lernen von und mit Lernenden gleichen Wissensstandes, den sog. Peers (Topping 2005). Eine Gruppe Lernender (mind. 2 Teilnehmer) lernt durch Interaktionen untereinander, bspw. in Form von Diskussionen (Jiang und Ting 2000). Durch diese Interaktionen werden bei Lernenden kognitive Prozesse 
Tab. 2 Gestaltungsrichtlinien für ein lernerzentriertes Flipped Classroom-Konzept

\begin{tabular}{|c|c|c|}
\hline Lernmethode & Designelemente (D) & Anforderung (A) \\
\hline \multirow[t]{7}{*}{ Peer Learning } & $\begin{array}{l}\text { D1) Gruppenbildung: Lernende zu einer Gruppe zusammenführen } \\
\text { und auf Gleichen Wissenstand achten }\end{array}$ & A1 \\
\hline & $\begin{array}{l}\text { D2) Aufgabenstruktur: Klare Definition einer mehrstufigen Auf- } \\
\text { gabenstellung mit aufeinander aufbauenden Teilaufgaben zur } \\
\text { kollaborativen Lösungsfindung }\end{array}$ & A1, A6 \\
\hline & $\begin{array}{l}\text { D3) Aufgabenformulierung: Formulierung offener Fragen mit } \\
\text { klaren Anweisungen wie die Lernenden miteinander interagieren } \\
\text { sollen }\end{array}$ & $\mathrm{A} 1, \mathrm{~A} 2, \mathrm{~A} 6, \mathrm{~A} 7$ \\
\hline & $\begin{array}{l}\text { D4) Dozent: Bereitstellung von Person mit didaktischem Know- } \\
\text { how zur Unterstützung und Begleitung der Lernenden. }\end{array}$ & A5 \\
\hline & $\begin{array}{l}\text { D5) Gegenseitigkeit: Bereitstellung von Aufgaben, damit Lernen- } \\
\text { de untereinander interagieren }\end{array}$ & $\mathrm{A} 2, \mathrm{~A} 6, \mathrm{~A} 7$ \\
\hline & $\begin{array}{l}\text { D6) Verantwortlichkeit: Verpflichtung von Lernenden zur Auf- } \\
\text { gabenbearbeitung durch sozialen Druck }\end{array}$ & A2, A6 \\
\hline & $\begin{array}{l}\text { D7) Ergebnissicherung: Richtigstellen von fehlerhaften Lösungen } \\
\text { sowie Vorstellen von Best-Practice-Lösungen }\end{array}$ & $\mathrm{A} 3, \mathrm{~A} 5$ \\
\hline \multirow[t]{2}{*}{ Peer Creation } & $\begin{array}{l}\text { D8) Ergebnisqualität: Integration von Feedback-Mechanismen, } \\
\text { um die Lösungen auszutauschen }\end{array}$ & $\mathrm{A} 1, \mathrm{~A} 2$ \\
\hline & $\begin{array}{l}\text { D9) Wissensbasis: Abgleich des Wissensstandes durch Überprü- } \\
\text { fung des Wissensstandes bevor Gruppen zusammenarbeiten }\end{array}$ & A6 \\
\hline \multirow[t]{2}{*}{$\begin{array}{l}\text { Peer } \\
\text { Assessment }\end{array}$} & $\begin{array}{l}\text { D10) Feedback: Erhalt von Feedback und Geben von Feedback } \\
\text { mithilfe vorgegebener Kriterien, um miteinander zu lernen }\end{array}$ & $\begin{array}{l}\text { A } 2, \text { A } 3, \text { A } 4, \text { A6, } \\
\text { A7 }\end{array}$ \\
\hline & $\begin{array}{l}\text { D11) Freitextaufgaben: Integration von Übungsaufgaben, die } \\
\text { das Bearbeiten komplexer Lerninhalte fordern, hohe kognitive } \\
\text { Lernziele adressieren und Rückmeldung zur individuellen Lern- } \\
\text { erfolgskontrolle liefern }\end{array}$ & $\mathrm{A} 2, \mathrm{~A} 6, \mathrm{~A} 7$ \\
\hline $\begin{array}{l}\text { Self } \\
\text { Assessment }\end{array}$ & $\begin{array}{l}\text { D12) Computergestützte Tests: Bereitstellung von Wissens- } \\
\text { tests mit automatisierter Auswertung zur eigenen, individuellen } \\
\text { Lernerfolgskontrolle }\end{array}$ & A3 \\
\hline
\end{tabular}

ausgelöst, Denkanstöße gegeben und die individuelle Reflexions-, Kooperations- und Kommunikationsfähigkeit gefördert (Arbaugh 2010; Oeste et al. 2014b). Darüber hinaus erfolgt eine tiefere Auseinandersetzung mit den Lerninhalten (Wegener und Leimeister 2012). Die Aktivitäten der Peers werden zumeist durch eine Person mit didaktischen Kenntnissen geleitet und überwacht (Harris 1998). Die Art der Formulierung von Übungsaufgaben und die Zusammenarbeit in der Gruppe sind sehr stark strukturiert (Bittner und Leimeister 2014). Der Dozent gliedert die Übungsaufgabe in aufeinander aufbauende Teilaufgaben. Eine Gruppe Lernender erarbeitet gemeinsam eine Lösung. Durch Präsentation aller Gruppenlösungen ergibt sich die Gesamtlösung zur Aufgabe (Büttner et al. 2012). Eine Ausprägung des Peer Learning ist das Peer Creation, bei dem Lernende in der Gruppe gemeinsam Lernmaterial erstellen. Die Besonderheit dabei ist, dass Lernende innerhalb der Gruppe die Rolle von Lehrenden einnehmen und sich durch bestimmte Feedback-Mechanismen beurteilen, um dadurch die Qualität der Lernmaterialien zu steigern (Wegener und Leimeister 2012). Im Gegensatz zum Peer Creation tritt beim Peer Assessment in der Regel der Lernende zunächst allein in Aktion. Nach Erstellung einer individuellen Leistung beurteilen sich dann die Lernenden untereinander und geben Feedback oder Bewertungspunkte entsprechend festgelegter Kriterien (Boud und Falchikov 2007). 
Dadurch werden sich Lernende mehr der eigenen Stärken und Schwächen bewusst und können ihre eigenen Leistungen, zumindest zum Teil, mit denen der Peers vergleichen und beurteilen (Darling-Hammond et al. 1995).

Damit liefern die verschiedenen Ausprägungsformen von Peer Learning wichtige Erkenntnisse zur didaktisch sinnvollen Ausgestaltung von lernerzentrierten Aktivitäten im Lernprozess. Lernende erarbeiten sich ihr Wissen selbst und können dieses durch Interaktionen mit anderen Lernenden erweitern, vertiefen und überprüfen. Durch Peer Learning wird somit eine intensive Auseinandersetzung mit Lerninhalten möglich, sodass auch in Massenlehrveranstaltungen hohe kognitive Lernzielebenen erreicht werden können.

Eine Möglichkeit, sich individuell mit den Lerninhalten auseinanderzusetzen und eine Rückmeldung auf die eigene Leistung zu erhalten, bietet das Self-Assessment. Computergestützte Tests erlauben die automatisierte Auswertung von Aufgabentypen, die durch eine Lösungsauswahl, wie bspw. Wahr/Falsch-Aussagen oder Multiple Choice (vgl. dazu Schiefner (2007), charakterisiert sind. Lernende erhalten eine sofortige, individuelle Rückmeldung, um ihren Lernstand entsprechend der kognitiven Lernzielebenen Erinnern, Verstehen und Anwenden selbst einzuschätzen (Bischof 2013). Computergestützte Tests können unabhängig von Ort und Zeit verwendet werden und erlauben dadurch flexibles Lernen. Zusätzlich wird der Dozent entlastet, der für die Überprüfung der Aufgaben keine Ressourcen benötigt (Bischof 2013).

Basierend auf den in Tab. 1 aus der Interaktionstheorie abgeleiteten Anforderungen, werden in der Tab. 2 die Designelemente (D) für ein Flipped Classroom-Konzept dargestellt. Das Peer Learning vereint $u$. a. die Ausprägungen von Peer Creation und Peer Assessment. Da diese nicht klar voneinander abzugrenzen sind, tauchen in der Tab. 2 Designelemente auf, die dem Peer Learning zugeordnet sind. Die Designelemente werden entsprechend der Zielvorgaben der einzelnen Lernmethoden formuliert. Zusätzlich wird bei jeder Gestaltungsrichtlinie die entsprechende Anforderung (A) aufgeführt, die damit adressiert wird.

\section{Lernerzentriertes Flipped Classroom-Konzept am Beispiel einer Wirtschaftsinformatik-Grundlagenveranstaltung}

Die Anwendung des Flipped Classroom-Konzepts erfolgt im Rahmen einer Wirtschaftsinformatik-Grundlagenveranstaltung, die von Bachelorstudierenden aus den Bereichen der Wirtschaftswissenschaften an einer deutschen Universität belegt wird. Diese Veranstaltung war bisher als traditionelle, dozentenzentrierte Vorlesung konzipiert, die jedes Semester von 150 bis 300 Studierenden besucht wurde. Zu den 12 Präsenzveranstaltungen gab es zusätzlich Tutorien, die in kleineren Gruppen abgehalten wurden. Über das universitäre Lernmanagementsystem (LMS ) Moodle standen den Studierenden Veranstaltungsskript und weiteres Lernmaterial zur Verfügung.

Im Folgenden wird die Anwendbarkeit der Gestaltungsrichtlinien für das Flipped Classroom-Konzept am Beispiel der Wirtschaftsinformatik-Grundlagenveranstaltung erläutert, die im letzten Sommersemester mit 250 Lernenden durchgeführt 


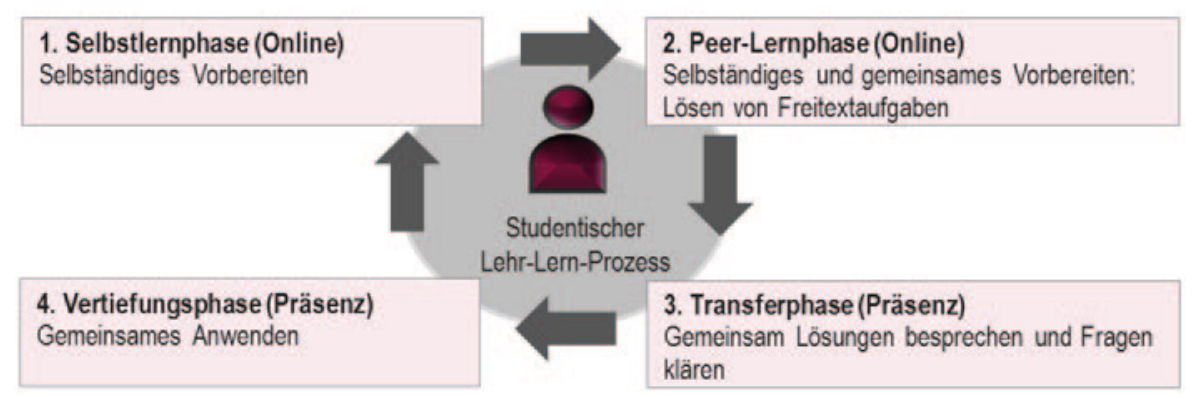

Abb. 1 Studentischer Lehr-Lern-Prozess der Wirtschaftsinformatik-Grundlagenveranstaltung im Flipped Classroom

wurde. Die folgende Abb. 1 zeigt den studentischen Lehr-Lern-Prozess im Flipped Classroom-Konzept mit vier aufeinander ablaufenden Phasen:

Der in der Abbildung dargestellte Lernzyklus hat eine Zeitdauer von zwei Wochen. Während eines Semesters wird der Lernzyklus fünf Mal wiederholt. Jeder Lernzyklus besteht aus vier Phasen, die nacheinander folgend ablaufen: Die Phase eins ist die Selbstlernphase und erfolgt im Selbststudium. Die Lerninhalte, die in traditionellen Lehrveranstaltungen in der Präsenzzeit vermittelt wurden, werden nun im Selbststudium mittels Lehrvideos und Lehrskript selbständig angeeignet. Zusätzlich stehen über das LMS Moodle der Universität diverse Übungsaufgaben im Antwort-WahlVerfahren als computergestützte Tests mit Sofort-Feedback bereit, um das Gelernte auf Wissen und Verständnis zu überprüfen. Für die zweite Phase, die Peer-Lernphase, stehen Freitextaufgaben zur Verfügung. Diese Freitextaufgaben sind sehr komplex, erfordern hohe kognitive Denkprozesse und adressieren die Lerninhalte auf den hohen Lernzielebenen. Gruppen bearbeiten online und kollaborativ über das LMS die Freitextaufgaben und bereiten eine gemeinsame Lösung in Form von Power Point Folien vor (Oeste et al. 2014b). Zu Semesterbeginn tragen sich die Lernende in einer von 12 Gruppen ein, in der sie auch das Tutorium gemeinsam besuchen. Jede Gruppe hat im LMS ein Forum, das für die gemeinsame Arbeit in der Peer-Lernphase verwendet wird. Einmalig fand in dieser zweiten Phase anstelle des kollaborativen Arbeitens in Gruppen das Peer Assessment statt (Lehmann et al. 2015). Anders als beim Bearbeiten der Freitextaufgaben in der Gruppe mussten diese individuell bearbeitet werden. Jede Lösung wurde über das LMS an die Peers per Zufall verteilt und von bis zu fünf Kommilitonen beurteilt. Lernende erhielten das Feedback mit Verbesserungsvorschlägen zurück und konnten die eigene Lösung, basierend auf dem Feedback, überarbeiten. Die Lösungen der Freitextaufgaben dienen dem Dozenten als essentieller Input für die dritte Phase. Über das LMS hat der Dozent Zugriff auf alle Einzel- bzw. Gruppenlösungen. Diese sog. Transferphase erfolgt in Präsenz und dient dazu, Fragen und Unklarheiten, die bei den Lernenden während der ersten beiden Phasen aufgetreten sind, in einer durch den Dozenten moderierten Diskussion zu klären. Darüber hinaus werden zu den Freitextaufgaben alle Gruppenlösungen bzw. verschiedene Einzellösungen durch den Dozenten vorgestellt sowie inhaltliche Stärken und Schwächen durch die Diskussion mit den Lernenden hervorgehoben. Die Vertiefungsphase erfolgt in Präsenz im Tutorium und behandelt lehrveranstaltungsrelevante Inhalte wie Daten- 
Tab. 3 Darstellung der einzelnen Phasen im Lernzyklus

\begin{tabular}{|c|c|c|}
\hline \multicolumn{2}{|c|}{ 1. Phase: Selbstlernphase (Online) } & \multirow{2}{*}{$\begin{array}{l}\text { Gestaltungsrichtlinien } \\
\text { D9, D12 }\end{array}$} \\
\hline$\overline{\text { Ziel }}$ & Selbstständiges Erarbeiten und Überprüfen der Lerninhalte & \\
\hline Interaktion & Lerner - Inhalte & \\
\hline Aufgabenspektrum & $\begin{array}{l}\text { Lerninhalte in Form von Lehrvideos, Lehrskripten, Lehr- } \\
\text { folien und computergestützten Übungsaufgaben }\end{array}$ & \\
\hline Ergebnis & $\begin{array}{l}\text { Lernende eignen sich Faktenwissen an und können Wis- } \\
\text { sen durch computergestützte Tests überprüfen, wodurch } \\
\text { eine individuelle Überprüfung des eigenen Lernstan- } \\
\text { des erfolgt. Nach Absolvierung der Phase haben alle } \\
\text { Lernenden ein gleiches Wissensniveau. Dies bildet die } \\
\text { Ausgangslage für die nächste Phase }\end{array}$ & \\
\hline Lernziele & Erinnern, Verstehen & \\
\hline \multicolumn{3}{|c|}{ 2. Phase: Peer-Lernphase (Online) } \\
\hline Ziel & $\begin{array}{l}\text { Anwendung und Austausch von Fakten- und } \\
\text { Anwendungswissen }\end{array}$ & $\begin{array}{l}\text { D1, D2, D3, D5, D6, } \\
\text { D8, D10, D11 }\end{array}$ \\
\hline Interaktion & Lerner - Lerner/Lerner - Inhalte & \\
\hline Aufgabenspektrum & $\begin{array}{l}\text { Lerninhalte in Form von komplexen Freitextaufgaben, } \\
\text { teils zur kollaborativen, teils zur individuellen Lö- } \\
\text { sungserarbeitung. IT-gestützte Diskussionen, Refle- } \\
\text { xionen und Feedback über das LMS unterstützen die } \\
\text { Lösungserarbeitung }\end{array}$ & \\
\hline Ergebnis & $\begin{array}{l}\text { Lösungen zu den Freitextaufgaben, die in der Gruppe } \\
\text { oder individuell erstellt wurden, werden auf Folien } \\
\text { vorbereitet und dienen als Input für die Phase } 3 \text {. Bei der } \\
\text { kollaborativen Arbeit erstellen die Gruppen die Lösungen } \\
\text { auf den Folien. Im Falle der individuellen Lösungserstel- } \\
\text { lung erstellt der Dozent die Folien }\end{array}$ & \\
\hline Lernziele & $\begin{array}{l}\text { Erinnern, Verstehen, Anwenden, Analysieren, Beurteilen, } \\
\text { Gestalten }\end{array}$ & \\
\hline \multicolumn{3}{|c|}{ 3. Phase: Transferphase (Präsenz) } \\
\hline Ziel & $\begin{array}{l}\text { Austausch, Verdeutlichung und Festigung von Fakten- } \\
\text { und Anwendungswissen }\end{array}$ & D3, D4, D7 \\
\hline Interaktion & Lerner - Dozent/Lerner - Lerner/Lerner - Inhalte & \\
\hline Aufgabenspektrum & $\begin{array}{l}\text { Darstellung der Lösungen durch Dozenten. Rückfragen } \\
\text { durch Lernende sowie Verinnerlichung der Lerninhal- } \\
\text { te und Lösen von Verständnisschwierigkeiten mittels } \\
\text { Diskussion }\end{array}$ & \\
\hline Ergebnis & $\begin{array}{l}\text { Verinnerlichung der Lerninhalte. Individuelle Überprü- } \\
\text { fung des eigenen Lernstandes }\end{array}$ & \\
\hline Lernziele & $\begin{array}{l}\text { Erinnern, Verstehen, Anwenden, Analysieren, Beurteilen, } \\
\text { Gestalten }\end{array}$ & \\
\hline \multicolumn{3}{|c|}{ 4. Phase: Vertiefungsphase (Präsenz) } \\
\hline Ziel & $\begin{array}{l}\text { Austausch, Verdeutlichung und Festigung von Fak- } \\
\text { ten- und Anwendungswissen im Rahmen verschiedener } \\
\text { Transferaufgaben }\end{array}$ & D1, D3, D4, D7 \\
\hline Interaktion & Lerner - Dozent/ Lerner - Lerner/ Lerner - Inhalte & \\
\hline Aufgabenspektrum & $\begin{array}{l}\text { Teilnahme an den Tutorien sowie Lösungserstellung und } \\
\text { Diskussionen mit anderen Lernenden und Dozent (Tutor) }\end{array}$ & \\
\hline Ergebnis & $\begin{array}{l}\text { Üben und Festigen der Lerninhalte durch Wissenstransfer } \\
\text { (Theorie - Praxis) }\end{array}$ & \\
\hline Lernziele & $\begin{array}{l}\text { Erinnern, Verstehen, Anwenden, Analysieren, Beurteilen, } \\
\text { Gestalten }\end{array}$ & \\
\hline
\end{tabular}


modellierung und Geschäftsprozessmanagement (BPMN), deren Inhalte per se einen größeren Übungscharakter benötigen.

Tabelle 3 zeigt ausführlich die einzelnen Phasen im Lernzyklus im Flipped Classroom-Konzept mit der spezifischen Interaktionsform nach Moore et al. (1989) und den adressierten Lernzielen nach Anderson et al. (2001). Darüber hinaus ist für jede Phase die von den Lernenden durchzuführende Aktivität (Aufgabenspektrum) und das Ergebnis jeder Phase in der Tabelle beschrieben.

\section{Diskussion des Flipped Classroom-Konzepts}

Während im vierten Kapitel die konkrete Implementierung in einer Grundlagenveranstaltung zur Wirtschaftsinformatik dargestellt wurde, wird nun die Eignung des Ansatzes kritisch diskutiert und reflektiert, um Implikationen für Theorie und Praxis abzuleiten. In einer zu Semesterende online durchgeführten Umfrage wurden neben quantitativen auch qualitative Daten erhoben. Konkret sollten die Studierenden die eigene Meinung zum Konzept und zu den eingesetzten Lernmethoden darstellen sowie den notwendigen Aufwand beurteilen. Die Daten wurden anonym erhoben. Diese werden im Folgenden anhand der Potentiale und Limitierungen des Konzepts dargestellt.

Kernaspekt des abgeleiteten Flipped Classroom-Konzepts ist die Adressierung von Interaktion über alle Interaktionsformen hinweg. Hierzu wurden Anforderungen formuliert, welche die einzelnen Interaktionsformen adressieren und in Designelemente überführt. Dabei hat sich während der Implementierung gezeigt, dass die eingesetzten Lernmethoden von Peer Learning, Peer Creation und Peer Assessments geeignet sind, um Interaktion in einem innovativen Lehr-Lern-Szenario zu fördern. Die folgenden Auszüge aus den studentischen Evaluationen geben deutliche Hinweise darauf, dass die eingesetzten Maßnahmen die gewünschten Effekte erzielt haben, wenngleich diese auch teilweise mit einem höheren Aufwand seitens der Studierenden verbunden sind.

„Der Austausch von verschiedenen Ideen und Gedankengängen mit meinen Kommilitoninnen. Dadurch hat sich meine Sichtweise verbessert. Ich konnte von verschiedenen Perspektiven aus denken. “

Durch diese Elemente war es möglich, die Interaktion vor der eigentlichen Präsenzphase zu ermöglichen, welches ein Grundprinzip von Flipped Classroom ist, um die Präsenzlehre zu einer Phase der reichhaltigen Interaktion von Lernenden und Dozent zu machen (Kim et al. 2014). Dennoch zeigen sowohl die Erfahrungen aus der Implementierung als auch andere Forschungsergebnisse (Strayer 2012; Kim et al. 2014), dass diese Vorbereitung nicht von allen Lernenden gleichermaßen wahrgenommen wird und dementsprechend die Interaktion in der Präsenzphase leidet.

„Zwar sind Formen wie Gruppenarbeiten und das Peer Assessment gut, damit man gleich das Thema lernt und mit dem Feedback weiß, ob man es auch verstanden hat. Jedoch sind solche Lernmethoden auch ziemlich zeitaufwendig, man hat auch noch andere Vorlesungen. " 
Daher kann es zielführend sein, Lernende für die Vorbereitung zu motivieren, beispielsweise durch Incentivierung der Aktivitäten und das explizite Kommunizieren des persönlichen Mehrwertes (Briggs 2006) in der Selbstlern- und der Peer-Lernphase. Auch das Erzeugen eines Wir-Gefühls in der Peer-Lernphase kann als motivationsfördernd wahrgenommen werden (Oeste et al. 2014c). Hier spielen aber auch persönliche wie kulturelle Unterschiede eine Rolle, welche in der künftigen, personalisierten Ausgestaltung eines solchen Lehrkonzepts aufgegriffen werden müssen (Janson et al. 2014). Die Erfahrungen haben gezeigt, dass die Erstellung der Lehrvideos sehr aufwändig ist. Dieser Aufwand rentiert sich langfristig, da die Videos nicht für jedes Semester neu gedreht werden müssen. Weiterhin beansprucht das Erstellen von Übungsaufgaben für die Selbstlern- und die Peer-Lernphase Zeit. Dies kann aber aus der Vorlesungszeit in die vorlesungsfreie Zeit vorgezogen werden. Da die Transferphase nicht wöchentlich stattfindet, wird dann in der Vorlesungszeit freie Zeit gewonnen.

Besonders positiv sind die Erfahrungen mit dem Flipped Classroom-Konzept, da vorgelagerte Interaktionen der Lernenden mit Lernmaterialien sowie der Lernenden untereinander stattfinden. Dieser Aspekt hilft insofern sehr gut, dass Lernende während des Lernprozesses sofort Feedback bekommen, ob Inhalte nicht verstanden worden. Somit lässt sich in der Transferphase gezielt auf Probleme der Lernenden eingehen. Das Konzept des sog. Just-in-Time-Teaching (Novak et al. 1999) hat sich als zielführend erwiesen, indem die Lernergebnisse von bereitgestellten Tests vor jeder Präsenzphase ausgewertet und dahingehend problematische Themenaspekte für die anschließende Diskussion ausgewählt werden. Diese Diskussion wird im vorliegenden Fall durch ein Classroom Response-System realisiert (Lehmann und Söllner 2014), um die Interaktion im Hörsaal zu unterstützen. Beispielsweise können so schwierige Fragen gemeinsam gelöst und diskutiert werden.

\section{„Das versucht wurde alle Studenten zu involvieren und sicherzustellen, dass jeder seinen Teil beiträgt. “}

Insgesamt zeigen die Erfahrungen, dass die Lehre im Flipped Classroom zielgerichteter abläuft, da auf inhaltliche Probleme und Verständnisschwierigkeiten frühzeitig im Lernprozess reagiert werden kann. In traditionellen Lehrveranstaltungen erkennt der Dozent Probleme erst bei der Klausurkorrektur und kann diese bei den Lernenden nicht mehr lösen. Dies wiederum kann sich auf nachfolgende Lehrveranstaltungen negativ auswirken, die auf den Grundlagen aufbauen.

Die IT-Unterstützung durch das Classroom Response-System und durch weitergehende Kollaborationswerkzeuge für die Interaktion in der Gruppe (Janson et al. 2014a) erlauben die anonyme Interaktion im Hörsaal und vermeidet einen Gesichtsverlust für die Lernenden. Denn es hat sich besonders in einer Einführungsveranstaltung gezeigt, dass die Lernenden am Anfang oftmals Hemmungen haben in großen Gruppen zu interagieren (Sims 2003).

Das Konzept des Flipped Classroom ist nicht ohne Limitierung. Ein wichtiger Punkt ist die notwendige IT-Unterstützung im Lernprozess. Diese ermöglicht dem Lernenden in der Selbst- und Peer-Lernphase das Lernen im eigenen Tempo sowie die asynchrone Interaktion mit den Peers. Gleichzeitig setzt diese massive IT-Unterstützung aber das Vorhandensein geeigneter Endgeräte beim Lernenden voraus. Im 
vorliegenden Fall konnten sich die Lernenden für die Dauer des Semesters ein Tablet oder Netbook ausleihen, um in den einzelnen Lernphasen die über die IT bereitgestellten Lernmethoden und -inhalte nutzen zu können. Zudem ist durch das notwendige Streaming von Lehrvideos eine geeignete Breitbandverbindung notwendig. Zwar ist der gesamte Campus durch W-Lan flächendeckend versorgt, allerdings gab es eine erhebliche Anzahl von Studierenden, welche als Pendler die Universität besuchen und daher nicht immer Breitbandinternet zur Verfügung haben (Roach 2014). Aus diesem Grund wurden die Lehrvideos auch als Download zur Verfügung gestellt, damit Studierende sich während ihrer begrenzten Aufenthalte auf dem Campus die Videos schnellstmöglich lokal speichern konnten. Hiermit verbunden ist die erhebliche IT-Unterstützung im Lernprozess, welche Lernende anfangs in der Nutzung überforderte (Janson et al. 2015). Hierzu gehörte beispielsweise das genutzte LMS, welches die Selbst- und Peer-Lernphase unterstützte.

„Ich finde, das neue Konzept sehr innovativ und prinzipiell sinnvoll. Gerade da es sehr mediengestützt ist (Moodle, Vorlesungsvideos), gibt es jedoch viele potentielle Fehlerquellen. "

Es erwies sich als zielführend den Lernenden am Anfang sehr große Hilfestellungen zu geben, beispielsweise durch Tutorials. Dieses in der Didaktik als Scaffolding erprobte Konzept sorgt dafür, dass die Lernenden am Anfang eines Kurses stark unterstützt werden, wobei diese Unterstützung im Kursverlauf zurückgenommen wird und der Lernende sich auf seinen selbstgesteuerten Lernprozess konzentrieren kann (Kim et al. 2014).

Abschließend ist aber allen Lehr-Lern-Szenarien gemein, dass die entscheidende Zielgröße immer der Lernerfolg ist (Janson et al. 2014c). Wenn die angesprochenen Aspekte dabei berücksichtigt werden, ist das Flipped Classroom-Konzept ein innovativer Ansatz, welcher auch in einer universitären Massenlehrveranstaltung die Adressierung sowohl niedriger als auch hoher Lernzielebenen ermöglicht. In Anlehnung an die Dienstleistungsforschung (Leimeister 2012), die Konzepte der IT-Unterstützung (Leimeister 2014) sowie der Kundenintegration werden zudem eine enorme Skalierbarkeit der universitären Lehre ermöglicht, welche reichhaltige Lehr-Lern-Szenarien schafft und damit der „Humanisierung“ der universitären Massenlehre abseits der vielgepriesenen MOOCs Rechnung trägt (Roach 2014).

\section{Zusammenfassung und Ausblick}

Der vorliegende Beitrag beschreibt die theorie-motivierte Gestaltung eines Flipped Classroom-Konzepts und führt auf, wie die Qualität der Lehre trotz einer großen Anzahl an Lernenden zeit- und ressourcenschonend gesteigert werden kann. Die Ergebnisse des Beitrages verdeutlichen, wie in einer Massenlehrveranstaltung Interaktion adressiert und Lernende in ihrem Lernprozess unterstützt werden können. Die Ergebnisse des Beitrages sind für Wissenschaftler, Dozenten und Trainer gleichermaßen von praktischer Relevanz, da sie Erkenntnisse liefern, mit welchen Lernmethoden Interaktion, Zusammenarbeit und Feedback zeit- und ressourcenschonend in einer Massenlehrveranstaltung adressiert werden können. Ausgehend von 
der Interaktionstheorie wurden Anforderungen an ein Flipped Classroom-Konzept entwickelt. Diese Anforderungen wurden durch Designelemente adressiert, welche übergreifende Methoden des Peer Learning nutzen. Diese Designelemente wurden im Rahmen einer Grundlagenveranstaltung der Wirtschaftsinformatik genutzt, um ein Flipped Classroom-Konzept zu implementieren. Dieses aus vier Phasen bestehende Lehr-Lern-Szenario ermöglicht durch IT-Unterstützung ein skalierbares Konzept, welches in Massenlehrveranstaltungen Interaktion und Lernerfolg auch auf hohen Lernzielebenen adressiert. Ausgehend von den Erkenntnissen der Implementierung ist das Flipped Classroom-Konzept ein gebrauchstaugliches Lehr-Lern-Szenario, welches in Forschung und Praxis weiter untersucht werden sollte. Künftiger Forschungsbedarf besteht dabei insbesondere in der Motivationsförderung und der Anreizgestaltung, um die kontinuierliche Mitarbeit im Sinne des selbstgesteuerten Lernens zu verbessern. Nur durch eine entsprechende Incentivierung und in der Folge durch die Vorbereitung der Lernenden ist das Flipped Classroom-Konzept von Erfolg gekrönt. Somit müssen künftige Forschungsarbeiten konkret untersuchen, wie sich Lernende für Gruppenarbeiten zur Lösungserstellung von Übungsaufgaben sowie für das Geben von qualitativ hochwertigem Feedback untereinander motivieren lassen. Weiterhin soll die Ausgestaltung der Lehrvideos im Hinblick auf die Interaktion verbessert werden. Es sollen Übungsaufgaben in die Lehrvideos integriert werden, so dass Lernende ihr Wissen direkt im Lehrvideo überprüfen können. Weiterer Forschungsbedarf geht in die Erstellung sogenannter Erklärvideos. Zum einen sollen komplexe Sachverhalte in kurzen Sequenzen grafisch dargestellt und erklärt werden. Zum anderen sollen Lernende in die Erstellung der Lehrvideos eingebunden werden, die dem Dozenten in dem Video Fragen zu Verständnis und komplexen Sachverhalten stellen. Aus praktischer Sicht wäre die Kombination von MOOC-Inhalten und Lernmethoden des Flipped Classroom denkbar, damit Dozenten mehr die Rolle eines Coachs im Lernprozess als die Rolle eines Inhaltslieferanten wahrnehmen.

\section{Literatur}

Alavi M, Marakas GM, Yoo Y (2002) A comparative study of distributed learning environments on learning outcomes. Inf Syst Res 13(4):404-415

Anderson L, Krathwohl D, Airasian PW, Cruikshank KA, Mayer RE, Pintrich P, Wittrock J (2001) A taxonomy for learning, teaching, and assessing: a revision of bloom's taxonomy of educational objectives. Addison Wesley Longmann, NY

Arbaugh J (2010) Online and Blended Business Education for the 21st Century. Current Research and Future Directions. Elsevier Science

Behringer N (2014) Flipped classroom: Der Unterricht wird auf den Kopf gestellt. wissensblitz (130)

Bischof L (2013) Die digitale ${ }^{\circledR}$ evolution? Chancen und Risiken der Digitalisierung akademischer Lehre

Bittner EAC, Leimeister JM (2014) Creating Shared understanding in heterogeneous work groups - Why it matters and how to achieve it. J Manage Info Syst 31(1):111-143

Bligh DA (1998) What's the Use of Lectures? Intellect books, Eastbourne

Boud D, Falchikov N (2007) Rethinking assessment in higher education: learning for the longer term. Routledge, Abingdon

Briggs RO (2006) On theory-driven design and deployment of collaboration systems. Int J Hum-Comput Stud 64(7):573-582

Bryant J, Heath R (2000) Human communication theory and research: concepts, contexts, and challenges. Lawrence Erlbaum Associates, Mahwah 
Büttner G, Warwas J, Adl-Amini K (2012) Kooperatives lernen und peer tutoring im inklusiven unterricht. Zeitschrift Inkl 1-2

Darling-Hammond L, Ancess J, Falk B (1995) Authentic assessment in action: studies of schools and students at work. Teachers College Press, New York

Fredericksen E, Pickett A, Shea P, Pelz W, Swan K (2000) Student satisfaction and perceived learning with online courses: Principles and examples from the SUNY learning network. J Asynchronous learn netw 4(2):7-41

Gagné ED, Yekovich CW, Yekovich FR (1993) The cognitive psychology of school learning. HarperCollins College, New York

Garrison DR, Kanuka H (2004) Blended learning: uncovering its transformative potential in higher education. Internet High Educ 7(2):95-105

Haggard S (2013) The maturing of the MOOC (Research No. 130). Department for Business Innovation and Skills- UK Goverment, London

Harris A (1998) Effective teaching: a review of the literature. School Leadersh Manag: Former School Organ 18(2):169-183

Janson A, Ernst S-J, Lehmann K, Leimeister JM (2014a) Creating awareness and reflection in a large-scale IS lecture - the application of a peer assessment in a flipped classroom scenario. Vortrag, 4th Workshop on Awareness and Reflection in Technology-Enhanced Learning (ARTEL 2014) to be held in the context of EC-TEL 2014, Graz, Austria

Janson A, Peters C, Leimeister JM (2014b) Der Weg zur effizienten Bereitstellung kultursensitiver Dienstleistungen - erste Schritte mittels systematischer Modularisierung. In: Dienstleistungsmodellierung 2014, Wien, Austria

Janson A, Söllner M, Bitzer P, Leimeister JM (2014c) Examining the effect of different measurements of learning success in technology-mediated learning research. In: International Conference on Information Systems (ICIS), Auckland, New Zealand

Janson A, Söllner M, Leimeister JM (2015) Determinanten und Effekte sinngetreuer Nutzung von Learning Management Systemen. In: Wirtschaftsinformatik (WI) Konferenz 2015, Osnabrück, Germany

Jiang M, Ting E (2000) A study of factors influencing students' perceived learning in a web-based course environment. Int J Educ Telecommun 6(4):317-338

Johnson L, Adams Becker S, Estrada V, Freeman A (2014) NMC horizon report: 2014 higher education edition. Deutsche Ausgabe. The New York Media Consortium, Austin

Keengwe J, Onchwari G, Oigara JN (2014) Preface. In: Jared K, Grace O, James NO (Hrsg) Promoting active learning through the flipped classroom model. IGI Global, Hershey, S xviii-xxi

Kerres M (2001) Neue Medien in der Lehre: von der projektförderung zur systematischen Integration. In: Das Hochschulwesen. Forum für Hochschulforschung,-praxis und-politik, S 38-44

Kim MK, Kim SM, Khera O, Getman J (2014) The experience of three flipped classrooms in an urban university: an exploration of design principles. Internet High Edu 22(0):37-50

Lehmann K, Söllner M (2014) Theory-driven design of a mobile-learning application to support different interaction types in large-scale lectures. Vortrag, European Conference on Information Systems (ECIS), Tel Aviv, Israel

Lehmann K, Söllner M, Leimeister JM (2015) Der Wert von IT-gestütztem Peer Assessment zur Unterstützung des Lernens in einer Universitären Massenlehrveranstaltung. In: Wirtschaftsinformatik (WI) Konferenz 2015, Osnabrück, Germany

Leidenfrost B, Strassnig B, Schabmann A, Carbon CC (2009) Verbesserung der Studiensituation für StudienanfängerInnen durch Cascaded Blended Mentoring. Psychologische Rundsch 60(2):99-106

Leimeister JM (2012) Dienstleistungsengineering und-management. Springer DE, Berlin

Leimeister JM (2014) Collaboration Engineering. IT-gestützte Zusammenarbeitsprozesse systematisch entwickeln und durchführen. Springer DE, Heidelberg

Martin FG (2012) Will massive open online courses change how we teach? Commun ACM 55(8):26-28

Metzger C, Schulmeister R (2004) Interaktivität im virtuellem Lernen am Beispiel von Lernprogrammen zur Deutschen Gebärdensprache. In: Mayer HO, Treichel D (Hrsg) Handlungsorientiertes Lernen und eLearning. Grundlagen und Praxisbeispiele. Oldenbourg-Verlag, München, S 265-297

Moore A, Masterson JT, Christophel DM, Shea KA (1996) College Teacher Immediacy and Student Ratings of Instruction. Commun Educ 45(1):29-39

Moore MG (1989) Editorial: Three types of interaction. Am J Distance Educ 3(2):1-7

Moore MG, Kearsley G (2011) Distance education: a systems view of online learning. Wadsworth Publishing Company, Belmont

Morgan N, Saxton J (1991) Teaching, questioning, and learning. Routledge, NY 
Novak GM, Patterson ET, Gavrin AD, Christian W, Forinash K (1999) Just in time teaching. Am J Phys 67(10):937-938

Oeste S, Lehmann K, Janson A, Leimeister JM (2014a) Flipping the IS classroom - theory-driven design for large-scale lectures. Vortrag, International Conference on Information Systems (ICIS), Auckland

Oeste S, Söllner M, Leimeister JM (2014b) Engineering peer-to-peer learning processes for generating high quality learningmaterials. In: International Conference on Collaboration and Technology (CRIWG), Santiago, Chile

Oeste S, Wegener R, Leimeister JM (2014c) Herausforderungen und Best Practices der E-Learning Einführung im Unternehmen. In: Multikonferenz Wirtschaftsinformatik (MKWI), Paderborn, Germany

Peffers K, Tuunanen T, Gengler CE, Rossi M, Hui W, Virtanen V, Bragge J (2006) The design science research process: a model for producing and presenting information systems research. In: Chatterjee $\mathrm{S}$, Hevner A (Hrsg) Proceedings of the first international conference on design science research in information systems and technology (DESRIST 2006), Claremont, California, S 83-106

Picciano AG (2002) Beyond student perceptions: issues of interaction, presence, and performance in an online course. J Asynchronous learn networ 6(1):21-40

Roach T (2014) Student perceptions toward flipped learning: new methods to increase interaction and active learning in economics. Int Rev Econ Educ 17:74-84

Schiefner M (2007) E-Assessment in der Lehrerinnen-und Lehrerbildung: what's new with the „E “. Beiträge zur Lehrerbildung 25(1):59-70

Sims R (2003) Promises of interactivity: aligning learner perceptions and expectations with strategies for flexible and online learning. Distance Educa 24(1):87-103

Strayer JF (2012) How learning in an inverted classroom influences cooperation, innovation and task orientation. Learning Env Res 15(2):171-193

Thurmond V, Wambach K (2004) Understanding interactions in distance education: a review of the literature. Intern J Instr Technol Distance Learning 1(1):9-26

Topping KJ (2005) Trends in peer learning. Educ Psychol 25(6):631-645

Wang MC, Haertel GD, Walberg HJ (1990) What influences learning? A content analysis of review literature. J Educ Res 84(1):30-43

Wegener R, Leimeister J (2012) Peer creation of e-learning materials to enhance learning success and satisfaction in an Information Systems Course. Vortrag, ECIS, Barcelona

Wulf J, Blohm I, Leimeister JM, Brenner W (2014) Massive open online courses. Bus Inf Syst Eng 6(2):111-114 